\title{
On Irrelevant Literals in Pseudo-Boolean Constraint Learning
}

\author{
Daniel Le Berre $^{1,2}$, Pierre Marquis ${ }^{1,2,3}$, Stefan Mengel $^{2,1}$ and Romain Wallon ${ }^{1,2}$ \\ ${ }^{1}$ Université d'Artois, Lens, France \\ ${ }^{2}$ CRIL, CNRS UMR 8188, Lens, France \\ ${ }^{3}$ Institut Universitaire de France \\ \{leberre, marquis, mengel, wallon\}@cril.fr
}

\begin{abstract}
Learning pseudo-Boolean (PB) constraints in PB solvers exploiting cutting planes based inference is not as well understood as clause learning in conflict-driven clause learning solvers. In this paper, we show that PB constraints derived using cutting planes may contain irrelevant literals, i.e., literals whose assigned values (whatever they are) never change the truth value of the constraint. Such literals may lead to infer constraints that are weaker than they should be, impacting the size of the proof built by the solver, and thus also affecting its performance. This suggests that current implementations of PB solvers based on cutting planes should be reconsidered to prevent the generation of irrelevant literals. Indeed, detecting and removing irrelevant literals is too expensive in practice to be considered as an option (the associated problem is NP-hard).
\end{abstract}

\section{Introduction}

Even though modern SAT solvers are known to perform well in practice on many industrial benchmarks, there exist instances which remain hard to solve, even for state-ofthe-art solvers [Järvisalo et al., 2012]. In particular, this is true for unsatisfiable formulae for which inconsistency can only be derived with an exponential number of resolution steps, e.g., for pigeonhole-principle formulae [Haken, 1985]. Many of those hard formulae require the solvers to be able to either detect and break symmetries or to "count" so as to generate short proofs [Benhamou and Sais, 1994; Devriendt et al., 2016; Metin et al., 2019]. This was an important motivation for the development of pseudo-Boolean (PB) reasoning [Roussel and Manquinho, 2009], which benefits from the expressiveness of $P B$ constraints (linear equations or inequations over Boolean variables) and from the strength of the cutting planes proof system [Gomory, 1958; Hooker, 1988; Nordström, 2015]. This proof system is in theory strictly stronger than the resolution proof system used in SAT solvers, as the former $p$-simulates the latter [Cook et al., 1987]: any resolution proof can be simulated by a cutting planes proof of polynomial size w.r.t. the size of the original proof. Yet, in practice, none of the current PB solvers uses the full power of the cutting planes proof system [Vinyals et al.,
2018]. Indeed, most of them are built on a specific form of this proof system, which can be viewed as a generalization of resolution [Hooker, 1988]. This allows to extend clausal inference to pseudo-Boolean inference, inheriting many of the techniques used in SAT solving [Dixon and Ginsberg, 2002; Chai and Kuehlmann, 2005]. In particular, the success of the conflict-driven clause learning architecture of modern SAT solvers [Marques-Silva and Sakallah, 1999; Moskewicz et al., 2001; Eén and Sörensson, 2004] motivated its generalization to PB problems: when a conflict is encountered (i.e., when a PB constraint becomes falsified), the cancellation rule is applied between the conflicting constraint and the reason for the propagation of some of its literals to infer a new conflicting constraint. Note that, contrary to what happens for resolution based solvers, the reason may need to be weakened before resolving to preserve the conflict. This operation is repeated until the inferred constraint propagates some of its literals. The constraint is then learned and a backjump is performed.

Over the years, many PB solvers implementing variants of the cutting planes proof system have been developed [Dixon and Ginsberg, 2002; Chai and Kuehlmann, 2005; Sheini and Sakallah, 2006; Le Berre and Parrain, 2010]. Recently, RoundingSat [Elffers and Nordström, 2018] introduced an aggressive use of the division and weakening rules (see Section 2 for details about cutting planes rules). However, since the first PB evaluation [Manquinho and Roussel, 2006], it has been observed that PB solvers are not as efficient as resolution based solvers, which can solve PB problems by encoding PB constraints into clauses [Een and Sörensson, 2006; Martins et al., 2014; Sakai and Nabeshima, 2015]. While PB solvers based on cutting planes perform generally well on specific classes of benchmarks, they fail to run uniformly well on all benchmarks [Elffers et al., 2018a]. This is partly due to the complexity of deciding when to use the rules of the cutting planes proof system, and of implementing their application efficiently. The initial trend has been to replace the application of the resolution rules by the generalized resolution rules [Hooker, 1988] during conflict analysis. However, this approach is not satisfactory because it is equivalent to resolution when applied to clauses, and requires a specific preprocessing to derive cardinality constraints [Biere et al., 2014; Elffers and Nordström, 2020]. This is why recent years have seen a renewed interest in PB solving and in the theory of cutting planes based inference [Elffers and Nordström, 2018; 
Vinyals et al., 2018; Elffers et al., 2018a; Gocht et al., 2019].

In this paper, we provide a new perspective regarding the desirable properties of the above proof systems. In particular, we show in Section 3 that cutting planes based inference may introduce irrelevant literals in the derived constraints, i.e., literals that have no effect on the truth value of the constraint in which they appear. Such literals seem inherent to the use of this proof system, as most of its rules may produce them, meaning that PB solvers have either to switch back to a proof system equivalent to the weaker resolution - which ensures to produce no irrelevant literals, but can only infer clauses - or to deal with these literals. Our main contribution is to show that, not only irrelevant literals are produced by the derivation rules used in current PB solvers, but these literals also contribute to the inference of constraints that are not as strong as they should be. Unfortunately, checking whether a literal is relevant is NP-complete [Crama and Hammer, 2011, Section 9.6], so in practice it would be unrealistic to remove irrelevant literals from all PB constraints derived by a PB solver so as to infer stronger constraints. As a workaround, we introduce in Section 4 an incomplete algorithm for detecting and removing these literals. We use it to show in Section 5 that irrelevant literals are produced in practice by two PB solvers, namely Sat4j [Le Berre and Parrain, 2010] and RoundingSat [Elffers and Nordström, 2018], which competed in the PB evaluation 2016. Our experiments also show that, for some instances, irrelevant literals may have an impact on the size of the proofs built by the solver, threatening its performance.

\section{Preliminaries}

We consider a propositional setting defined on a finite set of propositional variables $V$ which are classically interpreted. A literal $l$ is a propositional variable $v \in V$ or its negation $\bar{v}$. In this context, Boolean values are represented by the integers 1 (true) and 0 (false) and thus $\bar{v}=1-v$. $\models$ denotes logical entailment and $\equiv$ denotes logical equivalence.

\section{Pseudo-Boolean Constraints}

A pseudo-Boolean $(P B)$ constraint is an inequation of the form $\sum_{i=1}^{n} \alpha_{i} l_{i} \Delta \delta$, where $\alpha_{i}$ s and $\delta$ are integers, $l_{i}$ s are literals and $\Delta \in\{\leq,<,=,>, \geq\}$. Each $\alpha_{i}$ is called a weight or coefficient and $\delta$ is called the degree of the constraint. Any PB constraint can be normalized in linear time into (a conjunction of) constraints of the form $\sum_{i=1}^{n} \alpha_{i} l_{i} \geq \delta$ in which the coefficients and the degree are all positive integers. Thus, in the following, we assume that all PB constraints are normalized. A cardinality constraint is a PB constraint with all its coefficients equal to 1 , and a clause is a cardinality constraint with its degree equal to 1 .

\section{Inference Rules and Proof Systems}

The PB counterpart of the well-known resolution proof system is the cutting planes proof system [Gomory, 1958], which defines the following rules [Roussel and Manquinho, 2009].

Saturation. The PB constraint $\alpha l+\sum_{i=1}^{n} \alpha_{i} l_{i} \geq \delta$ where $\alpha>\delta$ is equivalent to $\delta l+\sum_{i=1}^{n} \alpha_{i} l_{i} \geq \delta$.

Weakening. The PB constraint $\alpha l+\sum_{i=1}^{n} \alpha_{i} l_{i} \geq \delta$ entails the constraint $\sum_{i=1}^{n} \alpha_{i} l_{i} \geq(\delta-\alpha)$.
Division. For any integer $\rho$, the constraint $\sum_{i=1}^{n} \alpha_{i} l_{i} \geq \delta$ entails the constraint $\sum_{i=1}^{n}\left\lceil\frac{\alpha_{i}}{\rho}\right\rceil l_{i} \geq\left\lceil\frac{\delta}{\rho}\right\rceil$. When each $\alpha_{i}$ is divisible by $\rho$, both constraints are equivalent

Multiplication. The PB constraint $\sum_{i=1}^{n} \alpha_{i} l_{i} \geq \delta$ is equivalent to $\sum_{i=1}^{n} \mu \alpha_{i} l_{i} \geq \mu \delta$ for any integer $\mu>0$.

Addition. The conjunction of the two PB constraints $\sum_{i=1}^{n} \alpha_{i} l_{i} \geq \delta$ and $\sum_{i=1}^{n} \alpha_{i}^{\prime} l_{i}^{\prime} \geq \delta^{\prime}$ entails the sum of both constraints, i.e., $\sum_{i=1}^{n} \alpha_{i} l_{i}+\alpha_{i}^{\prime} l_{i}^{\prime} \geq\left(\delta+\delta^{\prime}\right)$.

Cancellation. The conjunction of the two PB constraints $\alpha l+\sum_{i=1}^{n} \alpha_{i} l_{i} \geq \delta$ and $\alpha \bar{l}+\sum_{i=1}^{n} \alpha_{i}^{\prime} l_{i}^{\prime} \geq \delta^{\prime}$ entails $\sum_{i=1}^{n} \alpha_{i} l_{i}+\alpha_{i}^{\prime} l_{i}^{\prime} \geq\left(\delta+\delta^{\prime}-\alpha\right)$. Note that when both constraints are clauses, cancellation is equivalent to classical resolution [Hooker, 1988].

We denote by generalized resolution the proof system based on the cancellation and saturation rules and by cutting planes the proof system allowing unrestricted linear combinations of PB constraints and divisions. Each of these proof systems is refutationally complete [Hooker, 1988].

\section{Irrelevant Literals in PB Constraints}

A specific problem arising with general PB constraints but not with clauses or cardinality constraints that are neither tautological nor contradictory is the presence of irrelevant literals, which can be characterized using conditioning.

Definition 1 (Conditioning). Given a PB constraint $\chi$ and $a$ consistent term $\tau, \chi \mid \tau$ is the conditioning of $\chi$ by $\tau$, obtained by replacing each literal in $\chi$ by 1 if it appears in $\tau$, or by 0 if its opposite appears in $\tau$. The constraint is normalized by moving constants in the left hand side to the right hand side.

Definition 2 (Irrelevant literal). A literal $l$ is said to be irrelevant w.r.t. a constraint $\chi$ when $\chi|l \equiv \chi| \bar{l}$. Otherwise, $l$ is said to be relevant w.r.t. $\chi$ (we also say that $\chi$ depends on l). Equivalently, $l$ is irrelevant w.r.t. $\chi$ when flipping the value of $l$ in any model $M$ of $\chi$ cannot make it a counter-model of $\chi$.

In the following, when there is no ambiguity about which constraint is considered, we omit the constraint and simply say that $l$ is relevant or irrelevant.

The following proposition is an easy consequence of the definition of literal relevance.

Proposition 1. If there exists an irrelevant literal $l$ with coefficient $\alpha$ in a constraint $\chi$, then all literals $l^{\prime}$ having a coefficient $\alpha^{\prime} \leq \alpha$ in $\chi$ are also irrelevant.

Proof. Towards a contradiction, let $\chi$ be the constraint $\alpha l+$ $\alpha^{\prime} l^{\prime}+\sum_{i=1}^{n} \alpha_{i} l_{i} \geq \delta$ with $\alpha^{\prime} \leq \alpha$ and $l$ irrelevant. Suppose that $l^{\prime}$ is relevant. There exists a model $M$ of $\chi$ such that $M$ satisfies $l^{\prime}$ and flipping its value makes $M$ a counter-model of $\chi$. Let us note $M^{\prime}$ this counter-model. As $l$ is irrelevant, we can suppose w.l.o.g. that it is falsified by $M$, and thus by $M^{\prime}$. $M$ satisfies the constraint $\chi \mid\left(\bar{l} \wedge l^{\prime}\right) \equiv \sum_{i=1}^{n} \alpha_{i} l_{i} \geq \delta-\alpha^{\prime}(1)$, and so it is for $M^{\prime}$, because $M$ and $M^{\prime}$ coincide on $l_{i}$ s. As $l$ is irrelevant, flipping its value cannot make $M^{\prime}$ a model of $\chi$. Thus, $M^{\prime}$ does not satisfy $\chi \mid\left(l \wedge \bar{l}^{\prime}\right) \equiv \sum_{i=1}^{n} \alpha_{i} l_{i} \geq \delta-\alpha(2)$. However, because $\alpha^{\prime} \leq \alpha$, we have (1) $\models(2)$, which is incompatible with the fact that $M^{\prime} \models(1)$. 
Example 1. In the constraint $10 a+5 b+5 c+2 d+e+f \geq 15$, the literal $d$ is irrelevant, and so it is for the literals $e$ and $f$. In particular, this means that assigning these literals to any truth value preserves the semantics of the constraint. Thus, the three constraints $10 a+5 b+5 c+2 d+e+f \geq 15$, $10 a+5 b+5 c \geq 15$ and $10 a+5 b+5 c \geq 11$ are logically equivalent.

Observe that, even though the constraints are logically equivalent in the previous example, they are not equivalent over the reals (in this case, the second one is the stronger). Over the Booleans, the slack of a constraint [Chai and Kuehlmann, 2005] is a good heuristic indicator of its strength.

Definition 3 (Slack). The slack of a $P B$ constraint $\sum_{i=1}^{n} \alpha_{i} l_{i} \geq \delta$ is the value $\left(\sum_{i=1}^{n} \alpha_{i}\right)-\delta$.

Example 2. Let us consider the constraints in Example 1.

- $10 a+5 b+5 c+2 d+e+f \geq 15$ has slack 9 .

- $10 a+5 b+5 c \geq 15$ has slack 5 .

- $10 a+5 b+5 c \geq 11$ has slack 9 .

This value has long been used by PB solvers, as it allows to efficiently detect propagations and conflicts. In particular, all coefficients having a weight greater than the slack have to be satisfied. As such, the smaller the slack, the better the constraint from the solver viewpoint.

\subsection{Inference Rules Producing Irrelevant Literals}

Irrelevant literals seem inherent to PB reasoning. Indeed, all cutting planes rules may infer constraints containing irrelevant literals as long as they do not preserve equivalence, even if the constraints used to produce them do not contain any such literals, as shown by the following examples.

Weakening. Take the constraint $3 a+3 b+c+d \geq 4$, in which all literals are relevant. If this contraint is weakened on $d$, the resulting constraint $3 a+3 b+c \geq 3$ does not depend on $c$ anymore.

Division. Take the constraint $6 a+5 b+c \geq 6$. All its literals are relevant, but dividing it by 2 leads to the inference of the same constraint as above, i.e., $3 a+3 b+c \geq 3$.

Addition. Take the two constraints $4 a+3 b+3 c \geq 6$ and $3 b+2 a+2 d \geq 3$, which both depend on all their literals. Adding them produces the constraint $6 a+6 b+3 c+2 d \geq 9$, in which $d$ is irrelevant.

Cancellation. If we cancel out literal $e$ with the two constraints $4 b+3 \bar{e}+3 c+2 a \geq 6$ and $4 a+3 e+2 b+2 d \geq 6$, in which all literals are relevant, we again get the constraint $6 a+6 b+3 c+2 d \geq 9$.

\subsection{Artificially Relevant Literals in PB Solvers}

Because the rules presented in the previous section are widely used by PB solvers during their conflict analysis, these solvers have to deal with constraints containing irrelevant literals. In particular, the main issue arises when cutting planes rules are applied to these constraints: these rules may cause irrelevant literals to become relevant in the newly inferred constraint. When this occurs, we say that the literal has become artificially relevant. As we show below, this may happen in different circumstances.

\section{Generalized Resolution Based Solvers}

Let us consider a generalized resolution based PB solver, such as Sat $4 j$ [Le Berre and Parrain, 2010]. Suppose that a conflict occurs on $4 a+4 b+3 \bar{e}+3 g+3 h+2 i+2 j \geq 16$ (o). If the reason for propagating $e$ is $6 a+6 b+4 c+3 d+3 e+2 f \geq 10$, the conflict analysis is performed by applying the cancellation rule on $e$ between these two constraints. However, in some cases, the resulting constraint may not be conflicting anymore. To preserve the CDCL algorithm invariant, the reason of $e$ may need to be weakened, e.g., on $c$. This produces the constraint $6 a+6 b+3 d+3 e+2 f \geq 6(\diamond)$, in which $f$ is irrelevant. Note that $(\diamond)$ has slack 14 . Applying the cancellation rule between the conflicting constraint $(\circ)$ and $(\diamond)$ produces the constraint $10 a+10 b+3 d+3 g+3 h+2 f+2 i+2 j \geq 19(\star)$, in which $f$ has become artificially relevant.

\section{Division Based Solvers}

A recent improvement in PB solving is RoundingSat [Elffers and Nordström, 2018], which implements an aggressive use of the division and weakening rules during conflict analysis.

Let us consider the constraint $17 a+17 b+8 c+4 d+$ $2 e+2 f \geq 23$ in which all literals are relevant. Suppose that, during the search performed by RoundingSat, $c$ and $f$ are satisfied and all other literals are falsified by some propagations. This constraint is now conflictual: to analyze the conflict, RoundingSat resolves it against the reason for one of its falsified literals, e.g., the reason for $\bar{d}$. RoundingSat weakens the constraint on $f$, as it is not falsified and its coefficient (2) is not divisible by the coefficient of $d$ (4), giving the constraint $17 a+17 b+8 c+4 d+2 e \geq 21(\nabla)$. Observe that $e$ is now irrelevant and that $(\nabla)$ has slack 27 . When RoundingSat applies the division by 4 , the constraint becomes $5 a+5 b+2 c+d+e \geq 6(\Delta)$, in which all literals are relevant.

As pointed out by a reviewer, in RoundingSat, irrelevant literals produced after weakening a reason are always falsified by the current assignment. Indeed, suppose that the literal $l$ it propagates has coefficient $\alpha$. By construction, all remaining satisfied and unassigned literals have a coefficient that is divisible by $\alpha$, and thus that is greater than $\alpha$. As $l$ is propagated, it is necessarily relevant, and Proposition 1 tells us that this is also the case for these literals.

\subsection{Impact of Artificially Relevant Literals}

Artificially relevant literals in the constraints inferred by the solver may lead to infer constraints that are weaker than they could be if irrelevant literals were not there in the first place. Indeed, remember that, by definition, an irrelevant literal may be removed from the constraint. This can be achieved by locally assigning it to a truth value, as shown below.

\section{Removal by Weakening}

A first approach is assigning irrelevant literals to 1, i.e., applying the weakening rule to these literals. This approach may sometimes trigger the saturation rule, so that coefficients are kept small enough. This may have an impact on the solver efficiency, especially when arbitrary precision is required.

Let us consider the case of generalized resolution based solvers above. If the irrelevant literal $f$ is weakened away from $(\diamond)$, this constraint becomes $6 a+6 b+3 d+3 e \geq 4$, which is saturated into $4 a+4 b+3 d+3 e \geq 4\left(\diamond_{w}\right)$, which 
has slack 10. If this constraint is used in place of $(\diamond)$ when applying the cancellation with (०), one gets $8 a+8 b+3 d+$ $3 g+3 h+2 i+2 j \geq 17\left(\star_{w}\right)$, which is strictly stronger than the constraint $(\star)(a \wedge b$ is not an implicant of the constraint any longer).

However, this approach does not always allow to infer stronger constraints. Indeed, if we apply it to the RoundingSat example, $e$ is weakened away from $(\nabla)$, giving the constraint $17 a+17 b+8 c+4 d \geq 19\left(\nabla_{w}\right)$, which has slack 27 . When the division by 4 is applied, the constraint becomes $5 a+5 b+2 c+d \geq 5\left(\Delta_{w}\right)$. Observe that $c$ and $d$ are now irrelevant: the constraint is equivalent to $a+b \geq 1$, which is strictly weaker than the constraint we obtained previously.

\section{Simple Removal}

The second approach is assigning irrelevant literals to 0, i.e., removing them without modifying anything else on the constraint. This approach allows to strengthen the constraint over the reals, although it remains equivalent over the Booleans.

If we apply it to the RoundingSat example, it indeed allows to derive a stronger constraint: from $(\nabla)$, we now get $17 a+17 b+8 c+4 d \geq 21\left(\nabla_{r}\right)$, which has slack 25 . This constraint produces, after applying the division by 4 , the stronger constraint $5 a+5 b+2 c+d \geq 6\left(\Delta_{r}\right)$.

However, considering the example of generalized resolution based solvers, this approach removes $f$ from $(\diamond)$, giving $6 a+6 b+3 d+3 e \geq 6\left(\diamond_{r}\right)$, which has slack 12 . When applying the cancellation rule between this constraint and $(\circ)$, one gets $10 a+10 b+3 d+3 g+3 h+2 i+2 j \geq 19\left(\star_{r}\right)$, which is stronger than $(\star)$, but weaker than $\left(\star_{w}\right)$. In this case, the weakening based approach is better.

\section{Slack Based Approach}

The previous examples show that neither of the two removal approaches is stronger in every situation. We thus consider a case-by-case approach for deciding which one to choose. To this end, we consider the slack of the PB constraint as a heuristic. Indeed, the slack is subadditive: given two PB constraints, the constraint obtained by adding them has a slack that is at most equal to the sum of the slacks of the original constraints. Minimizing the slack of the constraint to choose will put a stronger upper bound on the slack of the constraints that will be derived later on.

\section{Eliminating Irrelevant Literals}

To evaluate the impact of irrelevant literals on PB solvers, we designed an approach for identifying and removing them from PB constraints. Since deciding whether a literal is relevant in a given PB constraint is NP-complete [Crama and Hammer, 2011, Section 9.6], in practice, it seems unrealistic to perform a relevance test for each literal of each constraint derived by the solver. We thus propose an incomplete but efficient algorithm for testing literal relevance.

For the sake of illustration, the following PB constraint $\chi$, in which we would like to decide whether $l$ is relevant, will be used as running example:

$$
\alpha l+\sum_{i=1}^{n} \alpha_{i} l_{i} \geq \delta
$$

Recall that $l$ is irrelevant if and only if $\chi|l \equiv \chi| \bar{l}$. Note that $\chi \mid l$ and $\chi \mid \bar{l}$ only differ in the degree, and that the degree of $\chi \mid \bar{l}$ is greater. Thus, clearly $\chi|\bar{l} \models \chi| l$, and the equivalence test boils down to checking whether:

$$
\sum_{i=1}^{n} \alpha_{i} l_{i} \geq \delta-\alpha \models \sum_{i=1}^{n} \alpha_{i} l_{i} \geq \delta
$$

Observe that this statement holds if and only if there is no interpretation of $\sum_{i=1}^{n} \alpha_{i} l_{i}$ equal to any number between $\delta-\alpha$ and $\delta-1$. Thus, checking that $l$ is irrelevant is equivalent to checking that there is no subset of $\alpha_{1}, \ldots, \alpha_{n}$ whose sum equals any of these numbers, i.e., solving an instance of the subset-sum problem for each of these inputs.

It is folklore that this can be done in time $O(n \delta)$ using dynamic programming [Cormen et al., 2009, Chapter 34.5] that is pseudopolynomial in the encoding size. However, in our context, both $n$ and $\delta$ may be very large, and it would be very inefficient to solve subset-sum on such inputs. As a workaround, we present an approach for solving subset-sum incompletely. Our detection algorithm needs to ensure that there is no solution to the considered subset-sum instance in order to correctly detect irrelevant literals, even though some of them may be missed. To this end, we introduce a detection algorithm based on solving subset-sum modulo a given positive integer $p$ (fixed for all applications of this algorithm). Since modular arithmetic is compatible with addition, one can ensure that, if there is a solution for the subset-sum problem with the original values, this solution is also a solution of the subset-sum problem considered modulo $p$.

As this procedure remains time consuming, one can also take advantage of Proposition 1 to reduce the number of checks to perform. This can be achieved by ordering the literals by ascending coefficients. Only one check per coefficient is required, and once a relevant literal is identified, there is no more irrelevant literal to remove.

Example 3. Take the constraint $12 a+6 b+6 c+2 d+2 e \geq 18$. If we want to check the relevance of e in this constraint, the multiset of coefficients to consider is $\{12,6,6,2\}$ (as $e$ is ignored for the purpose of the check).

First, let us consider $p=5$. The multiset of coefficients modulo $p$ is $\{2,1,1,2\}$. The set of all possible subset sums modulo 5 is thus $\{0,1,2,3,4\}$, and $e$ is wrongly detected as relevant, since there exists a subset sum equal to $2 \equiv 17$ mod 5. If we now consider $p=6$, the multiset of coefficients becomes $\{0,0,0,2\}$, and the possible subset sums modulo 6 are $\{0,2\}$. It is thus impossible to find any sum equal to either $4 \equiv 16 \bmod 6$ or $5 \equiv 17 \bmod 6$, so e is irrelevant.

As a consequence, $d$ can also be removed, since it has the same coefficient as e. Then, as $c$ is relevant, it is detected as such by our algorithm, which never gives the wrong answer for relevant literals. All remaining literals are thus relevant and the removal stops.

\section{Experimental Results}

This section provides experimental results showing to what extent irrelevant literals are present in the constraints inferred by PB solvers. We also give an attempt to evaluate their impact on the performance. 
To do so, we implemented the incomplete detection algorithm presented above in Sat4j [Le Berre and Parrain, 2010], and consider the whole set of decision benchmarks containing only small integers used in the PB evaluation since its very first edition [Manquinho and Roussel, 2006]. After some preliminary experiments, we chose 4547 as parameter $p$ of our incomplete subset-sum algorithm, and 500 as bound on the number of literals in the constraints to consider. These values have been chosen in a way that nearly all constraints in our experiments are treated efficiently, while the number of literals wrongly detected as relevant remains reasonable.

All experiments presented in this section have been run on a cluster equipped with quadcore bi-processors Intel XEON E5-5637 v4 (3.5 GHz) and $128 \mathrm{~GB}$ of memory, with a memory limit set to 64 GB.

\subsection{Production of Irrelevant Literals}

In order to see to what extent PB solvers produce irrelevant literals, we first use the set of benchmarks as input for both Sat4j's implementation of generalized resolution (Sat4j-CP) [Le Berre and Parrain, 2010] and RoundingSat [Elffers and Nordström, 2018], without modifying their conflict analysis. We chose these two solvers because, in the latest competition, no other solver implemented cutting planes based inference to solve PB instances (note that RoundingSat was called cdcl-cuttingplanes at that time). For these experiments, we let these solvers run for 5 minutes, and let them dump at most 100,000 constraints derived during their conflict analyses, which are then given to the detection algorithm we implemented in Sat4j. The constraints are dumped after the application of any rule that may introduce irrelevant literals. In the case of $S a t 4 j-C P$, irrelevant literals may be produced either after having applied the weakening operation on the reason, or after having applied the cancellation rule between the reason and the conflicting constraints. Regarding RoundingSat, irrelevant literals are always "hidden". Indeed, for efficiency reasons, the weakening and division rules are applied at the same time (we decoupled these two operations for our experiments, as time was not considered). However, by construction, if the weakening operation produces irrelevant literals, the following division, by ensuring that the pivot for the cancellation rule has a weight equal to 1 , will make all irrelevant literals artificially relevant (the pivot can never be irrelevant, and so do literals sharing the same coefficient at the end). As such, irrelevant literals produced in RoundingSat become systematically artificially relevant at the same step.

The results of these experiments are shown in Figures 1 and 2 with boxplots illustrating how many irrelevant literals were produced by the solver for each family. Each boxplot displays the quartiles with the horizontal bars and the estimated minimum and maximum with the vertical bars computed from the number of detected irrelevant literals in each instance of this family. Points represent outliers, which are instances for which the number of detected irrelevant literals is either below or above the estimated minimum or maximum, respectively. Because there are big differences between the families, boxplots are drawn using logarithmic scales. Also, the number of constraints in which irrelevant literals appear varies between the families: in some families, only few con-

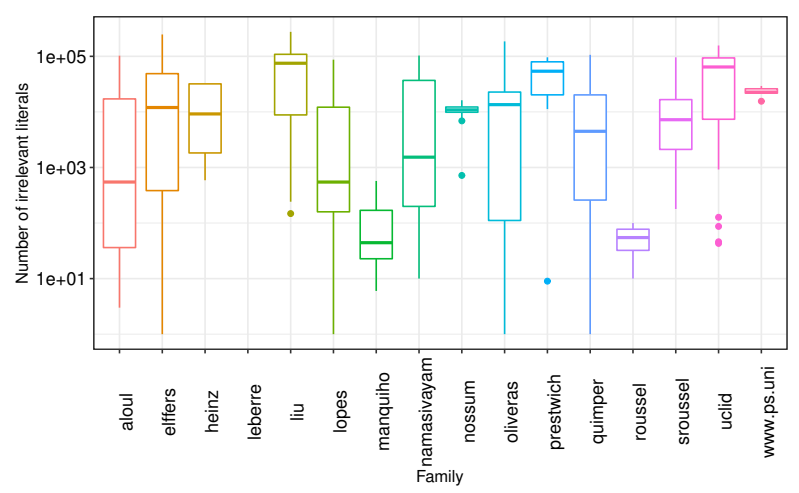

Figure 1: Number of irrelevant literals produced by $S a t 4 j-C P$. For readability, only submitters' names are displayed.

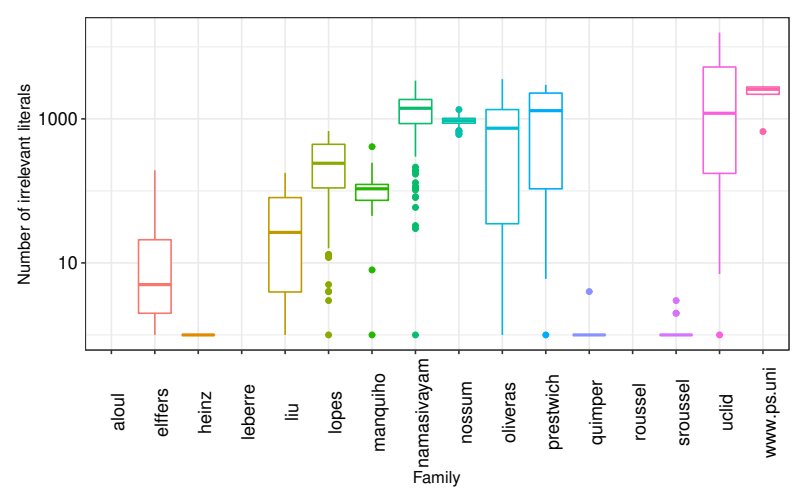

Figure 2: Number of irrelevant literals produced by RoundingSat. For readability, only submitters' names are displayed.

straints contain many irrelevant literals, whereas in some others up to $75 \%$ of the constraints contain irrelevant literals. These boxplots reveal that $S a t 4 j$ produces irrelevant literals (see Figure 1), and that this is also the case for RoundingSat, but to a lesser extent (see Figure 2). However, because all irrelevant literals produced by RoundingSat become immediately artificially relevant literals, they appear as irrelevant only once, while irrelevant literals produced by $S a t 4 j$ during conflict analysis may remain irrelevant in several consecutive derivation steps, and thus appear as such multiple times.

\subsection{Removal of Irrelevant Literals}

As we showed in a previous section, the presence of irrelevant literals in the constraints that are derived during conflict analysis may lead to the inference of weaker constraints. If we want to avoid this behavior, we need to remove all irrelevant literals produced during this process. Since this task is NP-hard, we used the incomplete approach (described above) for detecting these literals. However, this approach remains costly in practice, as $26 \%$ of the runtime is spent in average to detect irrelevant literals. For some families, such as Aardal_1, armies, ShortestPathBA and tsp, more than $75 \%$ of the runtime is required for most of the instances. This is why we considered a time independent measure to evaluate the impact of irrelevant literals: for all instances, we com- 


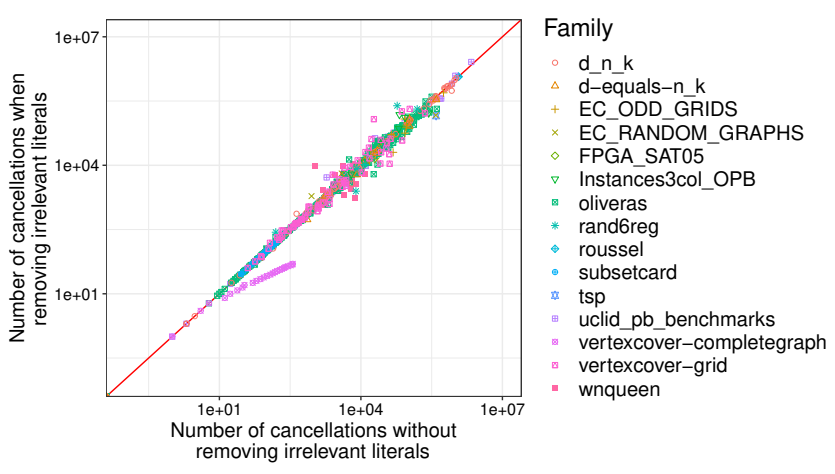

Figure 3: Comparison of the size of the proof built by Sat4j-CP on all unsatisfiable instances. The families used to describe the instances are the most specific subfamilies.

pared the sizes of the proofs built by the solver, measured in number of generalized resolution steps applied during the whole execution of the solver. Only unsatisfiable instances are considered here, as the size of the proofs only makes sense for such instances. The results are shown on Figure 3.

At first sight, it seems that there is not a great difference between the two approaches (this is also the case between the three different approaches presented in this paper for removing irrelevant literals, omitted for space reason). However, remember that our approach is incomplete and that, even though nearly all constraints are treated in most families (less than $75 \%$ of the constraints are treated only for instances from d-equals-n_k, FPGA_SATQ5, robin, ShortestPathBA and ShortestPathNG), we may wrongly detect as relevant literals that are actually irrelevant. To really evaluate the impact of irrelevant literals on the solver performance, we would need to remove all of them. However, in practice, a complete approach is clearly unreasonable: our algorithm manages to deal with constraints having a degree up to $10^{410415}$ which is out of reach of any complete approach.

Also, note that removing irrelevant literals impacts the heuristic used to select the variables to assign. Indeed, as for classical SAT solving, PB solvers use a heuristic based on VSIDS [Moskewicz et al., 2001]. This heuristic bumps the variables that are involved in a conflict, i.e., makes them more likely to be chosen next. When irrelevant literals are removed, the associated variables are not bumped anymore, which alters the behavior of the heuristic, and may have unexpected side effects as this heuristic is not fully understood [Elffers et al., 2018b]. In particular, it is hard to evaluate the impact of bumping irrelevant literals. On the one hand, one could argue that, because these literals are irrelevant, they do not play any role in the conflict. On the other hand, if they were relevant at some point, then their assignment may have triggered some propagations, and, in such a case, they may actually have contributed to the falsification of the constraint.

However, our contribution is not to find a solution to the problem of irrelevant literals, but to get a better understanding of their impact. To this end, let us consider more specifically the vertexcover-completegraph family for which Figure 3 shows that elimination of irrelevant literals has a significant impact on the size of the proof produced by $S a t 4 j$.
The instances of this family encode that complete graphs do not have small vertex covers [Elffers et al., 2018a]. As shown by Figure 3, the number of performed cancellations is exponentially smaller after removing irrelevant literals. A closer inspection at the solver's behavior shows that only few irrelevant literals are actually removed during the search. In particular, all these literals are detected and removed after the first conflict analysis, which produces a constraint of the form $k x_{1}+x_{2}+\ldots+x_{k} \geq k$, where $k=\left\lceil\frac{n}{2}\right\rceil-1$. One can observe that $x_{2}, \ldots, x_{k}$ are all irrelevant because their coefficients sum up to only $k-1$, and that the constraint is actually equivalent to the unit clause $x_{1} \geq 1$. In all further conflict analyses, no irrelevant literals are produced: this illustrates how few irrelevant literals may have an impact on the whole proof built by the solver, and may threaten its performance.

\section{Conclusion and Future Works}

In this paper, we have shown that irrelevant literals may be introduced in the constraints derived by PB solvers using cutting planes rules, and that such literals may have an impact on the strength of the reasoning. In particular, when irrelevant literals appear in the intermediate constraints, the learned constraint may be weaker than it could be, as it may contain artificially relevant literals. This may even happen when the constraint is a clause or a cardinality constraint, despite the fact that such constraints cannot contain irrelevant literals. We emphasized that while assigning irrelevant literals produces logically equivalent constraints, their slack may differ. The slack provides thus a convenient heuristic to handle irrelevant literals in a solver. To evaluate the practical impact of these literals on PB solvers, we designed an approximation algorithm for detecting and removing them at each derivation step. Our experimental results show that this approach allows to find irrelevant literals in PB solvers such as $S a t 4 j$ and RoundingSat, and that these literals may have an impact on the size of the proof they build.

Our approach for eliminating irrelevant literals is however too costly in practice to be considered as a counter-measure to their production in current PB solvers. A possible improvement for our algorithm, as suggested by one of the reviewers, is to consider multiple subset sum problems with small prime numbers, instead of one problem with a single large number, as for the Chinese remainder theorem. However, the best approach would be to avoid introducing irrelevant literals. Our ultimate goal is to define a proof system on PB constraints which ensures that constraints derived from PB constraints with only relevant literals do not contain irrelevant literals. The main difficulty is to find a complete set of rules that can be efficiently implemented to perform conflict analysis, and replace the current approaches used in PB solvers.

\section{Acknowledgements}

The authors are grateful to the anonymous reviewers for their numerous comments, that greatly helped to improve the presentation of the paper. Part of this work was supported by the French Ministry for Higher Education and Research and the Hauts-de-France Regional Council through the "Contrat de Plan État Région (CPER) DATA”. 


\section{References}

[Benhamou and Sais, 1994] Belaid Benhamou and Lakhdar Sais. Tractability Through Symmetries in Propositional Calculus. J. Autom. Reasoning, 12(1):89-102, 1994.

[Biere et al., 2014] Armin Biere, Daniel Le Berre, Emmanuel Lonca, and Norbert Manthey. Detecting Cardinality Constraints in CNF. In Proceedings of SAT'14, pages 285-301, 2014.

[Chai and Kuehlmann, 2005] Donald Chai and Andreas Kuehlmann. A fast pseudo-Boolean constraint solver. IEEE Trans. on CAD of Integrated Circuits and Systems, pages 305-317, 2005.

[Cook et al., 1987] William Cook, Collette R. Coullard, and György Turán. On the Complexity of Cutting-plane Proofs. Discrete Appl. Math., pages 25-38, 1987.

[Cormen et al., 2009] Thomas H. Cormen, Charles E. Leiserson, Ronald L. Rivest, and Clifford Stein. Introduction to Algorithms, Third Edition. The MIT Press, 2009.

[Crama and Hammer, 2011] Yves Crama and Peter L. Hammer. Boolean Functions: Theory, Algorithms, and Applications. Cambridge University Press, 2011.

[Devriendt et al., 2016] Jo Devriendt, Bart Bogaerts, Maurice Bruynooghe, and Marc Denecker. Improved Static Symmetry Breaking for SAT. In Proceedings of SAT'16, pages 104-122, 2016.

[Dixon and Ginsberg, 2002] Heidi E. Dixon and Matthew L. Ginsberg. Inference Methods for a Pseudo-Boolean Satisfiability Solver. In Proceedings of AAAI'02, pages 635640, 2002.

[Eén and Sörensson, 2004] Niklas Eén and Niklas Sörensson. An Extensible SAT-solver. In Proceedings of SAT'04, pages 502-518, 2004.

[Een and Sörensson, 2006] Niklas Een and Niklas Sörensson. Translating Pseudo-Boolean Constraints into SAT. JSAT, pages 1-26, 2006.

[Elffers and Nordström, 2018] Jan Elffers and Jakob Nordström. Divide and Conquer: Towards Faster PseudoBoolean Solving. In Proceedings of IJCAI'18, pages 1291-1299, 2018.

[Elffers and Nordström, 2020] Jan Elffers and Jakob Nordström. A Cardinal Improvement to Pseudo-Boolean Solving. In Proceedings of AAAI'20, 2020.

[Elffers et al., 2018a] Jan Elffers, Jesús Giráldez-Crú, Jakob Nordström, and Marc Vinyals. Using Combinatorial Benchmarks to Probe the Reasoning Power of PseudoBoolean Solvers. In Proceedings of SAT'18, pages 75-93, 2018.

[Elffers et al., 2018b] Jan Elffers, Jesús Giráldez-Cru, Stephan Gocht, Jakob Nordström, and Laurent Simon. Seeking Practical CDCL Insights from Theoretical SAT Benchmarks. In Proceedings of IJCAI'18, pages 1300-1308, 2018.

[Gocht et al., 2019] Stephan Gocht, Jakob Nordström, and Amir Yehudayoff. On Division Versus Saturation in
Pseudo-Boolean Solving. In Proceedings of IJCAI'19, pages 1711-1718, 2019.

[Gomory, 1958] Ralph E. Gomory. Outline of an algorithm for integer solutions to linear programs. Bulletin of the American Mathematical Society, pages 275-278, 1958.

[Haken, 1985] Armin Haken. The intractability of resolution. Theoretical Computer Science, pages 297-308, 1985.

[Hooker, 1988] John N. Hooker. Generalized resolution and cutting planes. Annals of Operations Research, pages 217239, 1988.

[Järvisalo et al., 2012] Matti Järvisalo, Daniel Le Berre, Olivier Roussel, and Laurent Simon. The International SAT Solver Competitions. AI Magazine, 33(1), 2012.

[Le Berre and Parrain, 2010] Daniel Le Berre and Anne Parrain. The SAT4J library, Release 2.2, System Description. JSAT, pages 59-64, 2010.

[Manquinho and Roussel, 2006] Vasco Manquinho and Olivier Roussel. The First Evaluation of Pseudo-Boolean Solvers (PB'05). JSAT, pages 103-143, 2006.

[Marques-Silva and Sakallah, 1999] Joao Marques-Silva and Karem A. Sakallah. GRASP: A Search Algorithm for Propositional Satisfiability. IEEE Trans. Computers, pages 220-227, 1999.

[Martins et al., 2014] Ruben Martins, Vasco Manquinho, and Inês Lynce. Open-WBO: A Modular MaxSAT Solver. In Proceedings of SAT'14, pages 438-445, 2014.

[Metin et al., 2019] Hakan Metin, Souheib Baarir, and Fabrice Kordon. Composing Symmetry Propagation and Effective Symmetry Breaking for SAT Solving. In Proceedings of NFM'19, pages 316-332, 2019.

[Moskewicz et al., 2001] Matthew W. Moskewicz, Conor F. Madigan, Ying Zhao, Lintao Zhang, and Sharad Malik. Chaff: Engineering an Efficient SAT Solver. In Proceedings of DAC'01, pages 530-535, 2001.

[Nordström, 2015] Jakob Nordström. On the Interplay Between Proof Complexity and SAT Solving. ACM SIGLOG News, pages 19-44, 2015.

[Roussel and Manquinho, 2009] Olivier Roussel and Vasco M. Manquinho. Pseudo-Boolean and Cardinality Constraints. In Handbook of Satisfiability, chapter 22, pages 695-733. IOS Press, 2009.

[Sakai and Nabeshima, 2015] Masahiko Sakai and Hidetomo Nabeshima. Construction of an ROBDD for a PB-Constraint in Band Form and Related Techniques for PB-Solvers. IEICE Transactions on Information and Systems, pages 1121-1127, 2015.

[Sheini and Sakallah, 2006] Hossein M. Sheini and Karem A. Sakallah. Pueblo: A Hybrid Pseudo-Boolean SAT Solver. JSAT, pages 165-189, 2006.

[Vinyals et al., 2018] Marc Vinyals, Jan Elffers, Jesús Giráldez-Crú, Stephan Gocht, and Jakob Nordström. In Between Resolution and Cutting Planes: A Study of Proof Systems for Pseudo-Boolean SAT Solving. In Proceedings of SAT'18, pages 292-310, 2018. 